TRANSACTIONS OF THE

AMERICAN MATHEMATICAL SOCIETY

Volume 362, Number 12, December 2010, Pages 6457-6476

S 0002-9947(2010)04978-5

Article electronically published on August 3, 2010

\title{
LINEARIZATION OF RESONANT VECTOR FIELDS
}

\author{
J. BASTO-GONÇALVES
}

\begin{abstract}
A method allowing the formal linearization of a large class of vector fields with resonant eigenvalues is presented, the admissible nonlinearities being characterized by conditions that are easy to check. This method also gives information on the terms that are actually present in a nonlinear normal form of a given resonant vector field.
\end{abstract}

\section{INTRODUCTION}

Normal forms for vector fields, or (autonomous) differential equations, are very important from the theoretical point of view, and also from the point of view of applications; in particular, they are the main technique in bifurcation theory, involving families of differential equations depending on parameters 2 .

The critical points of generic vector fields are never resonant, but the study of resonances becomes fundamental when considering families of vector fields, depending even on only one parameter.

Given a nonlinear vector field $X(x)=A x+a(x)$, where $a(x)$ is the nonlinear part, it follows from the classical results (section 2) that:

- If there are no resonance relations between the eigenvalues of $A$, the vector field is linearizable for any nonlinearity $a(x)$.

- If there are resonances, the vector field is reducible to a resonant normal form: its nonlinear part contains resonant monomials only.

Remark 1.1. If the nonlinear terms contain no resonant monomials, this does not mean that the corresponding vector field is linearizable 3 .

Remark 1.2. If the matrix $A$ is diagonalizable and the nonlinear terms contain only resonant monomials, or start with a resonant monomial, the corresponding vector field is not linearizable; however, this is not true if $A$ is not diagonalizable [4.

If there are resonances, the linearizability of the vector field depends on the monomials that are actually present in its nonlinear part: the linearizability of $X$ is not determined by its 1-jet (linear part) [11, in contrast to the classical linearization results and also those on topological equivalence [10, 7].

Our main objective here is, given a resonant matrix $A$ (i.e. with resonant eigenvalues), to present effective conditions on the nonlinearty $a(x)$ for the resonant vector field $X(x)=A x+a(x)$ to be formally linearizable and also to present a simple way of identifying the resonant monomials that have to appear in the normal

Received by the editors September 22, 2008.

2010 Mathematics Subject Classification. Primary 32S65, 34M35.

This research had financial support from the Fundação para a Ciência e a Tecnologia and the Calouste Gulbenkian Foundation.

(C)2010 American Mathematical Society Reverts to public domain 28 years from publication 
form of a given resonant vector field, in particular those of smaller degree, when holomorphic or $C^{\infty}$ linearization is impossible. This is especially important, as in many cases it permits us to establish the linearization with a finite degree of differentiability (Example 4.9).

The usual linearization procedure involves the resolution of a linear equation, the homological equation

$$
L_{A} h_{r}(c)=w_{r}(x)
$$

where $a(x)=w_{r}(x)+w_{r+1}(x)+\ldots$ and $w_{r}(x)$ is homogeneous of degree $r$, followed by a change of coordinates $x \mapsto x+h_{r}(x)$. This leads to a new nonlinearity $a^{\prime}(x)=w_{r+1}^{\prime}(x)+w_{r+2}^{\prime}(x)+\ldots$, and the process is repeated.

While it is straightforward to give conditions on a homogeneous component $w_{r}(x)$ so that the homological equation is solvable, it is harder to find conditions so that all subsequent homogeneous components after the changes of variables lead to solvable homological equations: even if there are no resonant monomials appearing in the vector field in the given coordinates, they can be created after some changes of coordinates in the process [3, example 1].

We look for conditions that guarantee the solvability of all the homological equations; by looking at the monomial $x^{K} e_{i}$ as a point $P_{i}^{K} \in \mathbb{Z}^{n}$, we obtain a geometric representation of the operations on the nonlinearity at each step, the basic fact being that the points corresponding to the monomials of the nonlinearity $a^{\prime}(x)$ are linear combinations with nonnegative integer coefficients of the points corresponding to the monomials in $a(x)$.

The results are presented in sections 3 and 4 and sections 6 and 7 describe the applications to vector field in two and three dimensions, respectively.

We restrict our considerations to the linearization problem in the formal category: in the holomorphic category, if the Brjuno condition is verified, the existence of a formal linearizing change of variables implies the existence of a holomorphic one [6]; in the smooth case, assuming hyperbolicity, the existence of a formal linearizing change of variables implies the existence of a $C^{\infty}$ linearization [9].

It is important to notice that all classical linearization results, in the analytic or in the $C^{\infty}$ category, are applicable (in the real case) only to hyperbolic critical points, as the absence of resonances implies hyperbolicity; under this condition, it follows from the Hartman-Grobman theorem that the vector fields are topologically conjugated to their linear parts.

The topological equivalence to the linear part in the resonant case was considered by Guckenheimer [10, for the Poincaré domain, Camacho et al. 7] (dimension 3) and Chaperon 8 for the Siegel domain under the condition that no two eigenvalues lie in the same line through the origin.

Thus our results can be applied to situations for which even the topological situation was not previously determined (subsections 6.2, 7.3, 7.6, 7.7).

We will consider our vector fields in complex variables, but the results are also valid for real vector fields; however, in that case they are effective essentially only when the eigenvalues are also real.

The approach presented here can be extended to diffeomorphisms instead of vector fields; Remark 5.3 presents an analogue of the main linearization result, applied in Example 5.6 to the 1-dimensional case. 


\section{BASIC RESULTS AND DEFINITIONS}

Let $X(x)$ be a holomorphic vector field on a domain $U$ in $\mathbb{C}^{n}$, with a singular point at the origin in $\mathbb{C}^{n}$ with linear part $A$ :

$$
X(x)=A x+a(x), \quad a(0)=\frac{\partial a}{\partial x}(0)=0 .
$$

It will always be assumed that $A$ is in the Jordan canonical form:

$$
A=\left[\begin{array}{cccccc}
\lambda_{1} & 0 & \cdots & \cdots & \cdots & 0 \\
\varepsilon_{1} & \lambda_{2} & \ddots & & & \vdots \\
0 & \varepsilon_{2} & \lambda_{3} & \ddots & \ddots & \vdots \\
\vdots & \ddots & \ddots & \ddots & \ddots & \vdots \\
\vdots & \ddots & \ddots & \ddots & \ddots & 0 \\
0 & \cdots & \cdots & 0 & \varepsilon_{n-1} & \lambda_{n}
\end{array}\right], \quad \varepsilon_{i} \in\{0,1\}, \varepsilon_{i}=1 \Rightarrow \lambda_{i}=\lambda_{i+1} .
$$

$X$ is said to be biholomorphically equivalent to its linear part if there exists a holomorphic change of coordinates $z=\psi(x)$, preserving the origin, $\psi(0)=0$, with inverse $x=\xi(z)$ also holomorphic, such that in the new coordinates the nonlinear part is zero:

$$
\frac{\partial \psi}{\partial x}(\xi(z)) X(\xi(z))=A z
$$

Let $\lambda=\left(\lambda_{1}, \ldots, \lambda_{n}\right) \in \mathbb{C}^{n}$ be the vector of the eigenvalues of the linear part $A$ of $X$, which are not assumed to be distinct (see [2]).

The eigenvalues are said to be resonant if, for some $i$, there exists $K=\left(k_{1}, \ldots, k_{n}\right)$, with $k_{j}$ nonnegative integers and $|K|=k_{1}+\cdots+k_{n}=k \geq 2$, such that

$$
\lambda_{i}=K \cdot \lambda \text {. }
$$

Then the number $k$ is called the order of this resonance. The monomial $x^{K} e_{i}$ is a resonant monomial attached to this resonance. Here and in what follows, $e_{i}$ denotes the vector whose coordinates are all zero except for the $i$-th one, which is 1 .

The vector $\lambda$ belongs to the Poincare domain if zero is not in the convex hull of the $n$ points $\left\{\lambda_{1}, \ldots, \lambda_{n}\right\}$ in the complex plane, and to the Siegel domain otherwise.

Poincaré Theorem ([2]). If the vector $\lambda$ belongs to the Poincaré domain and is nonresonant, $X$ is biholomorphically equivalent to its linear part in the neighbourhood of the singular point.

If $\lambda$ belongs to the Siegel domain, is not resonant and satisfies a Diophantine condition, $X$ is biholomorphically equivalent to its linear part in the neighbourhood of the singular point (Siegel theorem [2]). This theorem can be generalized.

A vector field with nonlinearity

$$
a(x)=\sum_{i=1}^{n} \sum_{|I| \geq 2} a_{i}^{I} x^{I} e_{i}
$$

satisfies the Brjuno $\omega$-condition if the series

$$
\sum_{k=1}^{\infty} \frac{1}{2^{k}} \ln \omega_{k}, \quad \omega_{k}=\min \left\{\left|I \cdot \lambda-\lambda_{i}\right|:\left|I \cdot \lambda-\lambda_{i}\right|>0,\left\|I-e_{i}\right\|<2^{k}\right\},
$$

is convergent. 
Brjuno Theorem ([6]). If $X$ is formally equivalent to its linear part and the Brjuno $\omega$-condition is verified, then $X$ is biholomorphically equivalent to its linear part in the neighbourhood of the singular point.

In the $C^{\infty}$ real case, if $\lambda$ is nonresonant (and therefore hyperbolic), $X$ is smoothly equivalent to its linear part in the neighbourhood of the singular point (Sternberg theorem, 13]), but we also have:

Chen Theorem ([9]). If $X$ is formally equivalent to its linear part and the singular point is hyperbolic, then $X$ is smoothly equivalent to its linear part in the neighbourhood of the singular point.

This theorem was improved in [5] to cover some nonhyperbolic cases, but the corresponding result is not applicable in our situation.

When there are resonances, the nonresonant terms can be eliminated by a formal change of variables, holomorphic under certain conditions:

Poincaré-Dulac Theorem ([2]). If the vector $\lambda$ belongs to the Poincaré domain, $X$ is biholomorphically equivalent, in the neighbourhood of the singular point, to a normal form consisting of its linear part and the resonant monomials.

The situation in the Siegel domain is much more difficult, as discussed in [6]: a very restrictive $A$-condition on the resonant normal form is involved in order to assure holomorphic equivalence.

\section{MAIN RESUlT}

Our aim is to obtain linearization results when there are resonances; this forces us to restrict the nonlinear terms: the linearizability of $X$ at the singular point $x_{0}$ in general is not determined by its 1-jet (linear part) [11, but the allowed nonlinearities are characterized by conditions that are easy to check.

Let $\mathbb{K}$ be the field of real numbers $\mathbb{R}$ or complex numbers $\mathbb{C}$, and denote by $\mathcal{F}=\mathbb{K}\left[\left[x_{1}, \ldots, x_{n}\right]\right]$ the formal power series algebra over $\mathbb{K}$. A formal vector field $X$ can be seen as a linear operator on $\mathcal{F}$, in fact as a derivation:

$$
X(f g)=X(f) g+f X(g), \quad f, g \in \mathcal{F} .
$$

As usual, we identify the set $\mathrm{D}(\mathcal{F})$ of derivations on $\mathcal{F}$ with $\mathcal{F}^{n}$ by writing

$$
X=\sum_{i=1}^{n} f_{i} \frac{\partial}{\partial x_{i}}, \quad f_{i} \in \mathcal{F}
$$

and, as we have been doing, we can consider $\frac{\partial}{\partial x_{i}}=e_{i}$.

In general terms, our main result (Theorem 3.4) can be described as follows: we are given a formal vector field $X(x)$ on a domain $U$ in $\mathbb{C}^{n}$, with a singular point at the origin in $\mathbb{C}^{n}$ with linear part $A$ :

$$
X(x)=A x+a(x), \quad a(x)=\sum_{i=1}^{n} \sum_{|K| \geq 2} a_{i}^{K} x^{K} e_{i} .
$$

This formal vector field can be the power series expansion of a holomorphic or a $C^{\infty}$ vector field.

To the linear vector field $A x$ we associate the linear operator $L_{A}$, the Lie derivative, defined on the vector space $\mathrm{D}(\mathcal{F})=\mathcal{F}^{\mathrm{n}}$ of formal vector fields. 
Let $\left(\lambda_{1}, \ldots, \lambda_{n}\right) \in \mathbb{C}^{n}$ be the eigenvalues of $A$. Then the eigenvalues of $L_{A}$ are all possible values $K \cdot \lambda-\lambda_{i}$ with $K=\left(k_{1}, \ldots, k_{n}\right)$ such that $k_{j}$ are nonnegative integers and $k_{1}+\cdots+k_{n} \geq 0$.

If the eigenvalues $\lambda$ of $A$ are resonant, $L_{A}$ has zero eigenvalues and it is not an isomorphism.

Given a nonlinearity $a(x)$, we can construct a subspace of $\mathrm{D}(\mathcal{F})$, or $\mathcal{F}^{n}$, generated (over $\mathbb{K}$ ) by its monomials:

$$
\mathfrak{A}_{\mathbb{K}}=\text { linear } \operatorname{span}_{\mathbb{K}} \mathfrak{A}, \quad \mathfrak{A}=\left\{x^{K} e_{i}, \text { such that } a_{i}^{K} \neq 0\right\} \subset \mathcal{F}^{n} .
$$

We want the restriction of $L_{A}$ to $\mathfrak{A}_{\mathbb{K}}$ to have the strong eigenvalue property 11 ; i.e. no integer (nonnegative) linear combination of its eigenvalues, $K \cdot \lambda-\lambda_{i}$ such that $a_{i}^{K} \neq 0$, is zero unless all coefficients are zero; in particular, zero is not an eigenvalue for that restriction.

The vector fields with such a nonlinearity $a(x)$ will be proved to be formally linearizable.

Definition 3.1. The nonlinearity $a(x)$ is admissible if all linear combinations with nonnegative integers (not all zero) of $Q_{i}^{K}=K \cdot \lambda-\lambda_{i}$, for which $a_{i}^{K}$ is nonzero, are nonzero.

This includes as a particular case the results obtained in [3], where all $K \cdot \lambda-\lambda_{i}$, for which $a_{i}^{K}$ is nonzero, were assumed to be positive integer multiples of a fixed nonzero complex number. A geometric interpretation of this hypothesis is that all points in the complex domain

$$
Q_{i}^{K}=K \cdot \lambda-\lambda_{i} \text { for which } a_{i}^{K} \neq 0
$$

belong to a straight (open) half-line through the origin and correspond to segments whose length is an integer multiple of a fixed nonzero number.

Let $\mathfrak{M}$ be the set of monomials in $\mathcal{F}^{n}$ :

$$
\mathfrak{M}=\left\{x^{K} e_{i}, k_{1}+\ldots+k_{n} \geq 0, i=1, \ldots, n\right\}
$$

and consider a representation of the monomial $x^{K} e_{i}$ by a point in $\mathbb{Z}^{n}$ :

$$
x^{K} e_{i} \mapsto P_{i}^{K}=K-e_{i}
$$

Let $\mathcal{M}$ be the set of points in $\mathbb{Z}^{n}$ such that at most one coordinate is -1 and all the others are nonnegative; $\mathcal{M}$ is the image of $\mathfrak{M}$ by the above representation.

The set $\mathcal{R}$ of points in $\mathcal{M}$ corresponding to resonant monomials in $\mathfrak{R}$ is characterized by

$$
\mathcal{R}=\{P \in \mathcal{M} \text { such that } \lambda \cdot P=0\} .
$$

Then, to a nonlinearity $a(x)$ and to the set $\mathfrak{A} \subset \mathfrak{M}$, there corresponds a set

$$
\mathcal{A}=\left\{P_{i}^{K}=K-e_{i} \text {, such that } a_{i}^{K} \neq 0\right\} \subset \mathcal{M} \subset \mathbb{Z}^{n} .
$$

We define $\mathcal{C}$ as the set of all linear combinations with nonnegative integers (not all zero) of points in $\mathcal{A}$ that belong to $\mathcal{M}$ (note that $-e_{i} \in \mathcal{M}$, but $-k e_{i} \notin \mathcal{M}$ for integer $k>1$ ), and $\hat{\mathcal{C}}=\lambda \cdot \mathcal{C}=\{\lambda \cdot P$ for $P \in \mathcal{C}\}$. The points $Q_{i}^{K} \in \mathbb{C}$ defined before verify $Q_{i}^{K}=\lambda \cdot P_{i}^{K} \in \hat{\mathcal{C}}$. 
Then $a(x)$ being admissible is equivalent to

$$
0 \notin \hat{\mathcal{C}}=\lambda \cdot \mathcal{C}, \text { i.e. } \lambda \cdot P \neq 0 \text { for any } P \in \mathcal{C}
$$

or

$$
\mathcal{C} \cap \mathcal{R}=\emptyset
$$

Let $\mathfrak{C}$ be the set of monomials whose corresponding points are in $\mathcal{C}$ :

$$
\mathfrak{C}_{\mathbb{K}}=\text { linear } \operatorname{span}_{\mathbb{K}} \mathfrak{C}, \quad \mathfrak{C}=\left\{x^{K} e_{i} \in \mathfrak{M} \text { such that } K-e_{i} \in \mathcal{C}\right\} .
$$

If the nonlinearity is admissible, the restriction of $L_{A}$ to $\mathfrak{C}_{\mathbb{K}}$ is an isomorphism. In fact it verifies the strong eigenvalue condition: the nonzero linear combinations with nonnegative integers of its eigenvalues are the elements of $\hat{\mathcal{C}}$.

Remark 3.2. We denote by the symbol $\mathfrak{S}$ a set of monomials, by the symbol $\mathcal{S}$ the set of vectors in $\mathbb{Z}^{n}$ that represent them under the map $x^{K} e_{i} \mapsto P_{i}^{K}=K-e_{i}$, and by $\hat{\mathcal{S}} \subset \mathbb{C}$ the set $\lambda \cdot \mathcal{S}$.

Example 3.3. Consider the vector field

$$
X(x, y)=\left(x+c_{1} x^{3} y,-y+c_{2} x y\right) .
$$

The eigenvalues of the linear part are $\lambda=(1,-1)$. The nonlinearity contains only the monomials $x^{3} y e_{1}$ and $x y e_{2} . \mathfrak{A}=\left\{x^{3} y e_{1}, x y e_{2}\right\}$; therefore it has all coefficients zero except $a_{1}^{(3,1)}=c_{1}$ and $a_{2}^{1,1}=c_{2}$, with

$$
P_{1}^{(3,1)}=(2,1), P_{2}^{(1,1)}=(1,0), \quad Q_{1}^{(3,1)}=Q_{2}^{(1,1)}=1 .
$$

Thus $\mathcal{A}=\{(2,1),(1,0)\}, \mathcal{C}=\{(2 n+m, n), n, m \in \mathbb{N}\} \cup\{\mathbb{N}(2,1)\} \cup\{\mathbb{N}(1,0)\} \subset \mathbb{Z}^{2}$, $\hat{\mathcal{C}}=\mathbb{N} \subset \mathbb{C} ;$ as $\mathcal{R}=\{(i, i), i \in \mathbb{N}\}$ we have

$$
\mathcal{C} \cap \mathcal{R}=\emptyset \text { and of course } 0 \notin \hat{\mathcal{C}},
$$

so the nonlinearity is admissible.

Theorem 3.4. Let $X$ be a formal (holomorphic, $C^{\infty}$ ) vector field on a neighbourhood $U$ of the origin in $\mathbb{C}^{n}$ which, in coordinates $x$, can be written as

$$
X(x)=A x+a(x), \quad a(0)=\frac{\partial a}{\partial x}(0)=0 .
$$

If the nonlinearity $a(x)$ is admissible (the Brjuno condition is verified, the critical point is hyperbolic), there exists a formal (holomorphic, $C^{\infty}$ ) change of coordinates $y=\psi(x)$ linearizing the vector field $X$.

By preventing resonances, all classical linearization results are applicable (in the real case) only to hyperbolic critical points. Under this condition, it follows from the Hartman-Grobman theorem that the vector fields are topologically conjugated to their linear parts. The above result can be applied to nonhyperbolic critical points in the holomorpic and real analytic categories, for which even the topological situation was not previously determined (subsections 6.2, 7.3, 7.6, 7.7).

Proof of Theorem 3.4. We follow the scheme for the proof of the existence of a formal linearization in [2], with small adaptations. We need to prove that the changes do not affect the conclusion, and mainly that, if we begin with an admissible nonlinearity, we only have admissible nonlinearities at every step. 
The vector field $X$ is written in the $x$ coordinates as

$$
X(x)=A x+a(x)=A x+\sum_{i=1}^{n} \sum_{|K| \geq 2} a_{i}^{K} x^{K} e_{i},
$$

where $a(x)$ is an admissible nonlinearity. By a convenient abuse of notation, we say that a monomial is admissible (for a given nonlinearity) if it belongs to $\mathfrak{C}$ or if it is represented by a point in $\mathcal{C}$.

We decompose $a(x)$ as

$$
a(x)=v_{r}(x)+v_{r+1}(x)+\ldots,
$$

where $v_{s}$ are the terms of degree $s \geq 2$. Furthermore, we write each $v_{r}$ as a sum of admissible monomials

$$
v_{r}(x)=\sum_{i=1}^{n} \sum_{|K|=r} a_{i}^{K} x^{K} e_{i}
$$

and order these monomials according to any given order that we take to be the lexicographical order on $(i, K)$. Denote by $m_{r}$ the first monomial in $v_{r}$, and let $w_{r}=v_{r}-m_{r}$. Then

$$
a(x)=m_{r}(x)+w_{r}(x)+v_{r+1}(x)+\ldots .
$$

If $A$ is diagonal, $A=\operatorname{diag}\left\{\lambda_{1}, \ldots, \lambda_{n}\right\}$, the solution $h$ of the homological equation

$$
L_{A} h(x)=m_{r}(x), \quad L_{A} h(x)=A h(x)-\frac{\partial h}{\partial x}(x) A x
$$

is a multiple of $m_{r}$ and therefore an admissible monomial. The change of coordinates $x=z+h(z)$ is also admissible $(h(z)$ is a monomial in $\mathfrak{C}$, represented by a point in $\mathcal{C}$ ), and it is easy to see that it gives, in the new coordinates $z$,

$$
X(z)=A z+w_{r}(z)+u_{r+1}(z)+u_{r+2}(z)+\ldots,
$$

where again $u_{k}$ stands for the terms of degree $k>r$.

The main point then is to prove that, in the new coordinates, the resulting nonlinearity is also admissible. This will follow from a sequence of lemmas, each corresponding to a step in the process of that change of coordinates $x=z+h(z)$; namely:

- Computing $a(z+h(z))$ : Lemma 3.5

- Computing the inverse $z=x+g(x)$ of $x=z+h(z)$ : Lemma 3.6.

- Computing $\left.\frac{\partial}{\partial x}(x+g(x))\right|_{x=z+h(z)} a(z+h(z))$ : Lemma 3.7

All operations on monomials will translate, under their representation as vectors in $\mathbb{Z}^{n}$, into linear combinations with nonnegative integer coefficients of the corresponding points in $\mathcal{M} \subset \mathbb{Z}^{n}$. As $\mathcal{C}$ is closed for those combinations, from its definition it follows that the nonlinearity will still be in $\mathfrak{C}_{\mathbb{K}}$ after the change of coordinates.

Lemma 3.5. If $x=z+a z^{I} e_{i}$ is a change of coordinates and $x^{J} e_{j}$ a monomial, such that the respective points $P_{i}^{I}=I-e_{i}$ and $P_{j}^{J}=J-e_{j}$ belong to $\mathcal{C}$, then the points corresponding to all monomials in $\left(z+a z^{I} e_{i}\right)^{J} e_{j}$ also belong to $\mathcal{C}$.

Proof. In fact all monomials in $\left(z+a z^{I} e_{i}\right)^{J} e_{j}$ are (ignoring coefficients) of the form

$$
z_{1}^{j_{1}} \ldots z_{i-1}^{j_{i-1}} z_{i}^{k} z^{\left(j_{i}-k\right) I} z_{i+1}^{j_{i+1}} \ldots z_{n}^{j_{n}}=z^{K} e_{j}, \quad K=J-j_{i} e_{i}+k e_{i}+\left(j_{i}-k\right) I .
$$


Thus

$$
K-e_{j}=J-e_{j}+\left(j_{i}-k\right)\left(I-e_{i}\right) \quad \text { or } \quad P_{j}^{K}=P_{j}^{J}+\left(j_{i}-k\right) P_{i}^{I},
$$

and therefore $P_{j}^{K}=K-e_{j} \in \mathcal{C}$.

Lemma 3.6. If $x=z+h(z)=z+a z^{I} e_{i}$ is a change of coordinates such that $P_{i}^{I}=I-e_{i} \in \mathcal{C}$, and its inverse is $z=x+g(x)$, then the points corresponding to all monomials in $g(x)$ also belong to $\mathcal{C}$.

Proof. Let

$$
g(x)=\sum_{j=1}^{n} \sum_{|J| \geq 2} g_{j}^{J} x^{J} e_{j} .
$$

Then, as $z=z+a z^{I} e_{i}+g\left(z+a z^{I} e_{i}\right)$, it follows that all monomials in $g\left(z+a z^{I} e_{i}\right)$ cancel each other leaving only $-a z^{I} e_{i}$ :

$$
\sum_{j=1}^{n} \sum_{|J| \geq 2} g_{j}^{J}\left(z+a z^{I} e_{i}\right)^{J} e_{j}=-a z^{I} e_{i} .
$$

Ordering the monomials by degree and lexicographic order on $(i, I)$ for the same degree, let $g_{j}^{J} x^{J} e_{j}$ be the first nonadmissible monomial in $g(x)$. From the previous lemma, we know that the admissible monomials in $g(x)$ give only admissible monomials in the variables $z$. On the other hand, $g_{j}^{J} x^{J} e_{j}$ gives rise to a nonadmissible monomial $g_{j}^{J} z^{J} e_{j}$, which cannot be cancelled: all monomials coming from lower order monomials are admissible, and all others are of higher order.

Therefore the set of nonadmissible monomials must be empty, and $g(x)$ is an admissible nonlinearity.

Alternatively, from $x=z+a z^{I} e_{i}$, it follows that $z_{j}=x_{j}$ for all $j \neq i$, and the remaining equation can be put in the form

$$
\xi=\eta+\alpha \eta^{k}, \quad \text { where } \alpha=a x^{I-i_{i} e_{i}}, \quad \xi=x_{i}, \quad \eta=z_{i}, \quad k=i_{i} .
$$

It is easy to see that this equation has a formal solution given by

$$
\begin{gathered}
\eta=\xi-c_{1}(k) \alpha \xi^{k}+c_{2}(k) \alpha^{2} \xi^{2 k-1}-c_{3}(k) \alpha^{3} \xi^{3 k-2}+\ldots, \\
c_{1}(k)=1, \quad c_{2}(k)=k, \quad c_{3}(k)=k^{2}+C_{2}^{k}, \quad \ldots
\end{gathered}
$$

when $k>0$ and by $\xi-\alpha$ when $k=0$ (this case is trivial). Thus we obtain $z=x+g(x)$, with

$$
\begin{aligned}
g(x) & =-a x^{I-i_{i} e_{i}} x_{i}^{i_{i}}+k a^{2} x^{2\left(I-i_{i} e_{i}\right)} x_{i}^{2 i_{i}-1}-c_{3}(k) a^{3} x^{3\left(I-i_{i} e_{i}\right)} x_{i}^{3 i_{i}-2}+\ldots \\
& =\sum_{r \geq 1} c_{r}(k) a^{r} x^{r\left(I-i_{i} e_{i}\right)+\left(r i_{i}-(r-1)\right) e_{i}} e_{i}=\sum_{r \geq 1} c_{r}(k) a^{r} x^{r\left(I-e_{i}\right)+e_{i}} e_{i} .
\end{aligned}
$$

The monomials in $g(x)$ are represented by points

$$
P_{i}^{r\left(I-e_{i}\right)+e_{i}}=r\left(I-e_{i}\right)=r P_{i}^{I} \in \mathcal{C}, \quad r \geq 1 .
$$

Lemma 3.7. Let $X$ be the vector field

$$
X(x)=A x+a(x), \quad a(0)=\frac{\partial a}{\partial x}(0)=0 .
$$


If the nonlinearity $a(x)$ is admissible, and if $x=z+\alpha z^{I} e_{i}$ is a change of coordinates with $P_{i}^{I}=I-e_{i} \in \mathcal{C}$, then, in the new coordinates $z$,

$$
X(z)=A z+b(z), \quad b(0)=\frac{\partial b}{\partial z}(0)=0,
$$

with $b(z)$ admissible.

Proof. We are assuming that $A=\operatorname{diag}\left\{\lambda_{1}, \ldots, \lambda_{n}\right\}$. Let $z=x+g(x)$ be the inverse of $x=z+\alpha z^{I} e_{i}$; from the previous lemma, Lemma 3.6, we know that $g(x)$ is admissible.

From $z=x+g(x)$ we obtain

$$
\begin{aligned}
\dot{z} & =\dot{x}+\sum_{j=1}^{n} \frac{\partial g(x)}{\partial x_{j}} \dot{x}_{j} \\
& =A x+a(x)+\sum_{j=1}^{n} \sum_{r=1}^{n} \sum_{|K| \geq 2} g_{r}^{K} k_{j} x^{K-e_{j}}\left(\lambda_{j} x_{j}+a_{j}(x)\right) e_{r} .
\end{aligned}
$$

The nonlinearity in the last member is admissible: $a(x)$ is admissible, as are the monomials $g_{r}^{K} k_{j} x^{K-e_{j}} \lambda_{j} x_{j} e_{r}=g_{r}^{K} k_{j} \lambda_{j} x^{K} e_{r}$. The monomials of $x^{K-e_{j}} a_{j}(x) e_{r}$ are also admissible, as they are of the form $x^{K-e_{j}} x^{J} e_{r}$, where $x^{J} e_{j}$ is admissible, and then

$$
\left(K-e_{j}+J\right)-e_{r}=\left(K-e_{r}\right)+\left(J-e_{j}\right) .
$$

We obtain $\dot{z}=A z+b(z)$ by using $x=z+\alpha z^{I} e_{i}$ in the last member of (3.1); the resulting nonlinearity $b(z)$ is admissible by Lemma 3.5 .

If $A$ has a nonzero nilpotent part, the solution of the homological equation ( $(\underline{H})$ is not necessarily a monomial multiple of $m_{r}(x)=x^{I} e_{i}$; i.e. $m_{r}(x)=x^{I} e_{i}$ is not necessarily an eigenvector of $L_{A}$ corresponding to the eigenvalue $\lambda \cdot I-\lambda_{i}$, it just belongs to its generalized eigenspace.

Thus, if $A$ is not diagonal (over $\mathbb{C}$ ), the solution of the homological equation $(\mathrm{H})$ is a polynomial $S_{r}(x)$, not necessarily a monomial. Then we make successive changes of coordinates based on the monomials involved in that solution. It follows from Lemma 3.8 that the end result is the same as far as the $r$-degree terms are concerned:

Lemma 3.8. Let $x=\xi_{1}(z)=z+\alpha z^{I} e_{i}$ and $x=\xi_{2}(z)=z+\beta z^{J} e_{j}$ be two diffeomorhisms around the origin, with $|I|=|J|=r$. Then the changes of coordinates $x=z+\alpha z^{I} e_{i}+\beta z^{J} e_{j}$ and $x=\xi_{1} \circ \xi_{2}(z)$ applied on the vector field $X(x)=A x+v_{r}(x)+v_{r+1}(x)+\ldots$, where $v_{s}$ are the homogeneous terms of degree $s \geq 2$, give the same $r$-degree terms.

Proof. The result follows from the fact that the $r$-degree terms obtained after a change of coordinates $x=z+h(z)$ are given by $v_{r}-L_{A}(h)$, and thus for the two changes of coordinates $x=z+\alpha z^{I} e_{i}+\beta z^{J} e_{j}$ and $x=\xi_{1} \circ \xi_{2}(z)$ the new $r$-degree terms are $v_{r}-L_{A}\left(\alpha z^{I} e_{i}+\beta z^{J} e_{j}\right)$ and $v_{r}-L_{A}\left(\alpha z^{I} e_{i}\right)-L_{A}\left(\beta z^{J} e_{j}\right)$, respectively.

We need to characterize the monomials appearing in $S_{r}(x)$, no longer just a multiple of $v_{r}(x)$.

Lemma 3.9. Let $z^{I} e_{i}$ and $z^{J} e_{j}$ be two monomials with corresponding points $P_{i}^{I}, P_{j}^{J}$ $\in \mathcal{M} \subset \mathbb{Z}^{n}$. Then the point corresponding to the monomials in $\left[z^{I} e_{i}, z^{J} e_{j}\right]$ is $P_{i}^{I}+P_{j}^{J}$. 
Proof. The two monomials in $\left[z^{I} e_{i}, z^{J} e_{j}\right]$ are, disregarding coefficients,

$$
z^{J-e_{i}} z^{I} e_{j}, \quad z^{I-e_{j}} z^{J} e_{i},
$$

and as

$$
P_{j}^{J-e_{i}+I}=P_{i}^{I-e_{j}+J}=J-e_{i}+I-e_{j}=P_{i}^{I}+P_{j}^{J},
$$

the lemma is proved.

Specializing Lemma 3.9 for the monomials in $A x$, we see that the monomials in $L_{A}\left(z^{I} e_{i}\right)$ are $z^{I} e_{i}$ or obtained from $z^{I} e_{i}$ (always disregarding coefficients) by some permutation of the type

$$
\begin{array}{ll}
z^{I} e_{i} \longrightarrow z^{I} e_{i+1}, & \varepsilon_{i}=1, \\
z^{I} e_{i} \longrightarrow z^{I-e_{j+1}+e_{j}} e_{i}, & \varepsilon_{j}=1,
\end{array}
$$

which, on the corresponding points, induce

$$
\begin{array}{ll}
P_{i}^{I}=I-e_{i} \longrightarrow P_{i+1}^{I}=I-e_{i+1}, & \varepsilon_{i}=1, \\
P_{i}^{I}=I-e_{i} \longrightarrow P_{i}^{I-e_{j+1}+e_{j}}=I-e_{j+1}+e_{j}-e_{i}, & \varepsilon_{j}=1,
\end{array}
$$

and it is easy to see that

$$
\lambda \cdot P_{i}^{I}=\lambda \cdot P_{i+1}^{I}, \quad \lambda \cdot P_{i}^{I}=\lambda \cdot P_{i}^{I-e_{j+1}+e_{j}} .
$$

Thus the monomials that appear in the solution $S_{r}(x)$ of the homological equation $L_{A}(x) h(x)=v_{r}(x)$ are such that the corresponding points are obtained from points in $\mathcal{C}$ by a permutation preserving the value of $\lambda^{*}$, the inner product by $\lambda$. Note that if we extend $\mathcal{C}$ to contain all these permutations, it is still true that $\lambda^{*}$ will be nonzero in the extended set.

Lemma 3.10. Lemma 3.7 remains valid for nonsemisimple linear parts.

Proof. If $A$ has a nonzero nilpotent part, the only change in the argument for the proof of Lemma 3.7 is that in (3.1) it appears that

$$
\sum_{j=1}^{n} \sum_{k=1}^{n} \sum_{|K| \geq 2} g_{k}^{K} k_{j} x^{K-e_{j}}\left(\varepsilon_{j} x_{j-1}+\lambda_{j} x_{j}+a_{j}(x)\right) e_{k}, \quad \varepsilon_{j}=0,1 .
$$

This gives new monomials $x^{K-e_{j}} x_{j-1} e_{k}$ when $\varepsilon_{j}=1$, and therefore $\lambda_{j-1}=\lambda_{j}$; the points $P_{k}^{K-e_{j}+e_{j-1}}$ representing those monomials again are obtained from points in $\mathcal{C}$ by a permutation preserving the value of $\lambda^{*}$, i.e. $\lambda \cdot P_{k}^{K-e_{j}+e_{j-1}}=\lambda \cdot P_{k}^{K}$, and therefore the argument can be extended to this case.

The only change made in the classical scheme of proof is that we solve the homological equation $(\underline{\mathrm{H}})$ for a monomial $m_{r}$ and not for the homogeneous term $v_{r}$. As the corresponding change of variables, or each one of a sequence of them corresponding to polynomial solutions of the homological equation $(\underline{\mathrm{H}})$, preserves $w_{r}=v_{r}-m_{r}$, our proof is complete since each step in the process leads to an admissible nonlinearity, in view of the lemmas. 


\section{VECTOR FIELDS WITH RESONANT MONOMIALS}

When $A$ is not semisimple, a monomial being resonant means that it belongs to the generalized eigenspace of the linear operator $L_{A}$ corresponding to the zero eigenvalue, but that monomial can still be in the image of $L_{A}$. Note that the possibility of solving the homological equation $(\underline{H})$ for a given monomial depends only on that monomial belonging to the image of $L_{A}$. These resonant monomials can be dealt with as long as they subsequently generate only monomials that belong to the image of the linear operator $L_{A}$.

We construct $\mathfrak{G}$ as a subset of the set $\mathfrak{R}$ of resonant monomials for which there exists another subset $\mathfrak{U} \subset \mathfrak{R}$ such that

$$
\mathfrak{G} \subset L_{A}(\mathfrak{U})_{\mathbb{K}}=\mathfrak{G}_{\mathbb{K}}, \quad[\mathfrak{G}, \mathfrak{U}] \subset \mathfrak{G},
$$

where $S_{\mathbb{K}}$ denotes the set of linear combinations with coefficients in $\mathbb{K}=\mathbb{R}, \mathbb{C}$ of elements of $S$.

The complement of $\mathfrak{G}$ in $\mathfrak{R}$ will be denoted by $\mathfrak{B}$. The sets $\mathfrak{G}, \mathfrak{U}$ and $\mathfrak{B}$ are not unique.

Remark 4.1. The zero monomial and the linear monomials $x_{i} e_{i}$ will always be considered in $\mathfrak{R}$ and in $\mathfrak{G}$, even if usually only the nonlinear monomials are explicitly stated.

Remark 4.2. The condition $L_{A}(\mathfrak{U})_{\mathbb{K}}=\mathfrak{G}_{\mathbb{K}}$ means that all monomials in $\mathfrak{G}$ can be obtained as the image by $L_{A}$ of a polynomial whose monomials are in $\mathfrak{U}$, and also that all monomials in $L_{A}(\mathfrak{U})$ belong to $\mathfrak{G}$.

Remark 4.3. It follows from Lemma 3.9 and Remark 3.2 that the condition $\mathcal{G}+\mathcal{U} \subset$ $\mathcal{G}$ follows from $[\mathfrak{G}, \mathfrak{U}] \subset \mathfrak{G}$.

As seen before, to a nonlinearity $a(x)$ there corresponds a set:

$$
\mathcal{A}=\left\{P_{i}^{K}=K-e_{i}, \text { such that } a_{i}^{K} \neq 0\right\} \subset \mathcal{M} \subset \mathbb{Z}^{n} .
$$

We extend $\mathcal{A}$ to a set $\mathcal{A}_{\text {ext }}$ so that:

- $\mathcal{A} \subset \mathcal{A}_{\text {ext }}, \quad \mathcal{A}_{\text {ext }}+\mathcal{U} \subset \mathcal{A}_{\text {ext }}$.

- $\mathcal{A}_{\text {ext }}$ is closed for the following permutations, whenever the resulting vector belongs to $\mathcal{M}$ (corresponds to some monomial):

$$
\begin{aligned}
& P_{i}^{K}=K-e_{i} \in \mathcal{A}_{\text {ext }}, \varepsilon_{i}=1 \Longrightarrow P_{i+1}^{K} \in \mathcal{A}_{\text {ext }}, \\
& P_{i}^{K}=K-e_{i} \in \mathcal{A}_{\text {ext }}, \varepsilon_{r}=1 \Longrightarrow P_{i}^{J} \in \mathcal{A}_{\text {ext }}, J=K-e_{r+1}+e_{r}
\end{aligned}
$$

or, in terms of monomials:

$$
\begin{aligned}
& x^{K} e_{i} \in \mathfrak{A}_{e x t}, \varepsilon_{i}=1, i<n \Longrightarrow x^{K} e_{i+1} \in \mathfrak{A}_{e x t}, \\
& x^{K} e_{i} \in \mathfrak{A}_{e x t}, \varepsilon_{r}=1, k_{r+1} \geq 1 \Longrightarrow x_{r} x_{r+1}^{-1} x^{K} e_{i} \in \mathfrak{A}_{e x t} .
\end{aligned}
$$

We define $\mathcal{C}$ as the set of all those linear combinations with nonnegative integers (not all zero) of vectors in $\mathcal{A}_{\text {ext }}$ that belong to $\mathcal{M}$.

Definition 4.4. A nonlinearity $a(x)$ is weakly admissible if all resonant monomials in $\mathcal{C}$ are in $\mathcal{G}$ :

$$
\mathcal{C} \cap \mathcal{R} \subset \mathcal{G} \text { or } \mathcal{C} \cap \mathcal{B}=\emptyset .
$$

Remark 4.5. The nonlinearity $a(x)$ is admissible, as in (A) , if it is weakly admissible, and $\mathfrak{G}$, and therefore $\mathcal{G}$, are empty and $\mathcal{B}=\mathcal{R}$. 
Example 4.6. Consider the vector field

$$
X(x, y, z)=(x, x+y, 2 z)+a(x, y, z) .
$$

We have $\lambda=(1,1,2)$ and $\mathfrak{R}=\left\{x^{2} e_{3}, x y e_{3}, y^{2} e_{3}\right\}$. Since

$$
L_{A}\left(x^{2} e_{3}\right)=0, \quad L_{A}\left(x y e_{3}\right)=x^{2} e_{3}, \quad L_{A}\left(y^{2} e_{3}\right)=2 x y e_{3},
$$

we see that we can choose

$$
\mathfrak{U}=\left\{x y e_{3}, y^{2} e_{3}\right\}, \quad \mathfrak{G}=\left\{x^{2} e_{3}, x y e_{3}\right\}, \quad \mathfrak{B}=\left\{y^{2} e_{3}\right\},
$$

since $[\mathfrak{U}, \mathfrak{G}]=\{0\}$ (see Remark 4.1). Then $a(x, y, z)$ is weakly admissible if

$$
a_{3}^{(0,2,0)}=0, \text { or equivalently } \frac{\partial^{2}}{\partial y^{2}} a_{3}(0,0,0)=0 .
$$

Theorem 4.7. Let $X$ be a formal (holomorphic, $C^{\infty}$ ) vector field on a neighbourhood $U$ of the origin in $\mathbb{C}^{n}$ which, in coordinates $x$, can be written as

$$
X(x)=A x+a(x), \quad a(0)=\frac{\partial a}{\partial x}(0)=0 .
$$

If the nonlinearity $a(x)$ is weakly admissible (the Brjuno condition is verified, the critical point is hyperbolic), there exists a formal (holomorphic, $C^{\infty}$ ) change of coordinates $y=\psi(x)$ linearizing the vector field $X$.

It is important to notice that this method also provides information on the normal form of the vector field $X$ when it is not formally linearizable:

Corollary 4.8. Let $X$ be a formal (holomorphic, $C^{\infty}$ ) vector field on a neighbourhood $U$ of the origin in $\mathbb{C}^{n}$ which, in coordinates $x$, can be written as

$$
X(x)=A x+a(x), \quad a(0)=\frac{\partial a}{\partial x}(0)=0 .
$$

$A$ resonant normal form for $X$ can be obtained involving only the nonlinear resonant monomials in $\mathfrak{C} \cap \mathfrak{B}$ corresponding to points in $\mathcal{C} \cap \mathcal{B}$.

Example 4.9. Let $X(x, y)=\left(x+a y^{4},-y+b x^{2}\right)$ be a vector field in $\mathbb{C}^{2}$. The eigenvalues are 1 and -1 , therefore resonant, the nonlinearity does not contain resonant monomials, but $\mathcal{C} \cap \mathcal{R}$ contains the points corresponding to the monomials of the form $x^{7 k+1} y^{7 k} e_{1}$ or $x^{7 k} y^{7 k+1} e_{2}$. Thus we see that in its normal form,

$$
X(x, y)=\left(x+x^{8} y^{7} \varphi\left(x^{7} y^{7}\right),-y+x^{7} y^{8} \psi\left(x^{7} y^{7}\right)\right),
$$

the lowest nonlinear terms are at least of degree 15.

Then we can apply the results of Sell [12] (see also the result of Samovol 1]) to prove $C^{7}$-linearization: the change of coordinates to obtain the above normal form is holomorphic, in fact polynomial, and we can take $Q=14$ (see [12]), noticing that there are no resonances of that order and all derivatives up to order 13 of the nonlinearity $F(x, y)=x^{7} y^{7}\left(x \varphi\left(x^{7} y^{7}\right), y \psi\left(x^{7} y^{7}\right)\right)$ are zero at the origin, as required.

Remark 4.10. The resonant normal form can be further simplied in many cases 14 , 15. The changes of coordinates then do not necessarily correspond to monomials in the image of $L_{A}$, they can correspond to monomials in $[\mathfrak{R}, \mathfrak{R}]$. 
Proof of Theorem 4.7. It follows from the lemmas that a monomial $z^{J} e_{j} \in \mathcal{C}$ gives rise, after a change of coordinates $x=z+z^{I} e_{i}$, to monomials corresponding to $\left(J-e_{j}\right)+s\left(I-e_{i}\right)$ or $\left(J-e_{j}\right)+\left(s\left(I-e_{i}\right)-e_{k}+e_{k-1}\right)$ when $\varepsilon_{k}=1$ in the canonical Jordan form.

Assume $v_{r}(x)$ is not resonant. Then all the monomials $z^{I} e_{i}$ in the solution $S_{r}(x)$ of the corresponding homological equation are in $\mathfrak{C}$, and the proof follows as for Theorem 3.4.

Only the case $v_{r}(x) \in \mathfrak{C} \cap \mathfrak{R} \subset \mathfrak{G}$ remains to be considered; then all monomials in $S_{r}(x)$ belong to $\mathfrak{U}$. The corresponding changes of coordinates are of the type $x=z+z^{I} e_{i}$, with $z^{I} e_{i} \in \mathfrak{U}$ but not necessarily $z^{I} e_{i} \in \mathfrak{C}$. Even so, it follows from the definition of the set $\mathcal{C}$ that it contains $\left(J-e_{j}\right)+s\left(I-e_{i}\right)$, as $\mathcal{C}+\mathcal{U} \subset \mathcal{C}$, and also $\left(J-e_{j}\right)+\left(s\left(I-e_{i}\right)-e_{k}+e_{k-1}\right)$, as $\mathcal{C}$ is closed for this type of permutation.

\section{Finite Determinacy}

In this context, $k$-determinacy means that the conditions involve only the $k$-jet of the vector field; see [11] for a very careful discussion of the subject.

In the Poincaré domain the number of possible resonances, and thus of resonant monomials, is finite. Denote by $d$ the biggest degree of a resonant monomial.

Corollary 5.1. Let $X$ be a (holomorphic, $C^{\infty}$ hyperbolic) vector field with the eigenvalue vector $\lambda$ in the Poincaré domain, such that

$$
j^{d} X(x)=A x+h_{d}(x),
$$

where $h_{d}(x)$ is a weakly admissible nonlinearity with terms of degree at most $d$. Then $X$ is linearizable.

Proof. The linearization procedure we have been describing works for the vector field $Y(x)=j^{d} X(x)=A x+h_{d}(x)$, giving a change of coordinates that linearizes $j^{d} X(x)$.

Making that change of coordinates in $X$ results in a new vector field $Z(x)=$ $A x+a(x)$. All monomials in the nonlinear part $a(x)$ of $Z$ are of the type $x^{I} e_{i}$ with $|K|>d$; therefore they can never be resonant or generate resonant vector fields at subsequent steps.

In the Siegel domain the linearizable resonant vector fields have infinite codimension and are never finitely determined. But if we consider special classes of vector fields, the situation can be completely different; in particular, if we consider polynomial vector fields of degree less than $r$, say, the codimension becomes finite, of course, and we can estimate it, as the next example shows:

Example 5.2. Consider a polynomial vector field of degree less than $r$ :

$$
X(x, y)=\left(x+a_{1}(x, y, x), x+y+a_{2}(x, y, x),-z+a_{3}(x, y, x)\right) .
$$

From the results of subsection 7.4 it follows that we need to avoid the monomials for whose corresponding points $\lambda^{*}<0$ or $\lambda^{*}=0, \mu^{*} \leq 0$ with $\mu=(0,-1,1)$; of course, instead of $\lambda^{*}<0$ we could choose to avoid $\lambda^{*}>0$.

If we consider quadratic nonlinearities, $r=3$, we see that there are no resonant monomials of degree 2 , but we have to avoid $z^{2} e_{1}$ and $y z e_{1}$ corresponding to the 
points $(-1,0,2)$ and $(-1,1,1), x z e_{1}, y z e_{2}$ and $z^{2} e_{3}$ corresponding to the point $(0,0,1), z^{2} e_{2}$ corresponding to the point $(0,-1,2), x z e_{2}$ corresponding to the point $(1,-1,1)$, as for these points $\lambda^{*}<0$. Thus the linearizable nonlinearities have codimension at most 7 in the space of all quadratic nonlinearities of dimension 18 .

If we also consider cubic nonlinearities, $r=4$, we see that we also have to avoid:

- $z^{3} e_{1}$ corresponding to the point $(-1,0,3), y z^{2} e_{1}$ corresponding to the point $(-1,1,2), x z^{2} e_{1}, y z^{2} e_{2}$ and $z^{3} e_{3}$ corresponding to the point $(0,0,2), x z^{2} e_{2}$ corresponding to the point $(1,-1,2), z^{3} e_{2}$ corresponding to the point $(0,-1,3)$, as for these points $\lambda^{*}<0$

- $y^{2} z e_{1}$ corresponding to the point $(-1,2,1), x^{2} z e_{2}$ corresponding to the point $(2,-1,1)$, and $x y z e_{1}, y^{2} z e_{2}$ and $y z^{2} e_{3}$ corresponding to the point $(0,1,1)$, as $\lambda=0, \mu \leq 0$, for these points.

Thus the linearizable nonlinearities have codimension at most 19 in the space of all quadratic and cubic nonlinearities.

Remark 5.3 (Linearization of maps). Let $F(x)$ be a holomorphic map with linear part $A$ (in the Jordan canonical form):

$$
F: U \subset \mathbb{C}^{n} \longrightarrow \mathbb{C}^{n}, \quad F(x)=A x+a(x), \quad a(0)=\frac{\partial a}{\partial x}(0)=0 .
$$

Definition 5.4. If $A$ is nonsingular, all its eigenvalues $\lambda=\left(\lambda_{1}, \ldots, \lambda_{n}\right) \in \mathbb{C}^{n}$ are nonzero. We define $\nu_{i}=\log \lambda_{i}$, and the nonlinearity $a(x)$ is admissible if all linear combinations with nonnegative integers (not all zero) of $K \cdot \nu-\nu_{i}$, for which $a_{i}^{K}$ is nonzero, are nonzero modulo $2 \pi i$.

Theorem 5.5. Let $F(x)=A x+a(x)$ be a formal (holomorphic, $C^{\infty}$ ) diffeomorphism on a neighbourhood $U$ of the origin in $\mathbb{C}^{n}$. If the nonlinearity a $(x)$ is admissible, there exists a formal change of coordinates $y=\psi(x)$ linearizing the map $F$.

Example 5.6. If $n=1$ and a map $X(x)=\lambda x+\sum_{i=2}^{\infty} a_{i} x^{i}$ is given, with $\lambda \neq 0$, then the nonlinearity is admissible if

$$
\left\{(k-1) \log \lambda, a_{k} \neq 0 \text { and } k \geq 2\right\} \cap(2 \pi i) \mathbb{Z}=\emptyset .
$$

Thus, if $\lambda$ is not a root of unity, all nonlinearities are admissible and all maps are formally linearizable. If $\lambda$ is a root of unity, no nonlinearity is admissible; our theorem says nothing about this situation.

\section{Applications: Vector fields in $\mathbb{R}^{2}$}

Example 6.1. The situation for dimension one is very simple: if a vector field $X(x)=\lambda x+\sum_{i=2}^{\infty} a_{i} x^{i}$ is given on $\mathbb{R}$, then

$$
\mathcal{M}=\{j \in \mathbb{Z}, j \geq-1\}, \quad \mathcal{A}=\left\{i-1, a_{i} \neq 0 \text { and } i \geq 2\right\},
$$

and clearly $\mathcal{C} \subset\{j \in \mathbb{Z}, j \geq 1\}$.

Therefore, if $\lambda \neq 0$, we have $0 \notin \lambda \cdot \mathcal{C}$, and all nonlinearities are admissible and all vector fields are formally linearizable. On the other hand, if $\lambda=0$, we have 
$0 \in \lambda \cdot \mathcal{C}$, and no nonlinearity is admissible; our theorem says nothing about this situation.

The Jordan canonical of the linear part of a resonant vector field in $\mathbb{R}^{2}$ is diagonal (over $\mathbb{C}$ ), unless the two eigenvalues are zero. That case is very similar to the case of vector fields in $\mathbb{R}^{3}$ with a Jordan block of dimension two, considered in the next section.

Here and in the next section we normalize the eigenvalues, using the fact that a linearizing change of coordinates for a vector field also linearizes any scalar multiple of that vector field.

The case of pure imaginary eigenvalues is then equivalent to the case of subsection 6.3 over $\mathbb{C}$, but over $\mathbb{R}$ it is much harder, and our results are not directly very useful.

The presence of resonances in the plane (real or complex) means that the condition for 1-determinacy of the topological situation [10, 7] is not verified, and our results are therefore useful even at that level. Finite determinacy is valid only in the case in subsection 6.1, but in any case we get (holomorphic, analytic, smooth) conjugacy to the linear part, not just topological equivalence.

In the plane and in the presence of resonances, the points $\lambda \cdot I-\lambda_{i}$ always belong to some straight line and (after normalization) are integers on the real line. The difference between Theorem 3.4 and the result of [3] is just this: in [3] those points have to be all positive or all negative, and now it is possible to allow the two signs as long as the linear combinations giving zero are not realizable as monomials, as shown in Example 6.2.

6.1. $\lambda=(1, k \neq 1), \quad k \in \mathbb{N}$. The only resonant monomial is $x^{k} e_{2}$. Therefore a vector field $X(x, y)=(x, k y)+a(x, y)$ will be linearizable if

$$
(k,-1) \notin \mathcal{C} ;
$$

otherwise its normal form will contain just one resonant monomial, exactly $x^{k} e_{2}$.

Example 6.2. Consider an arbitrary vector field with linear part $A=\operatorname{diag}(1, k)$ :

$$
X(x, y)=\left(x+a_{1}(x, y), k y+c_{2} x^{2}+\ldots+c_{k} x^{k}+a_{2}(x, y) y+a_{3}(x) x^{k+1}\right) .
$$

To be able to apply the Poincaré-Dulac theorem to get a linear normal form we need

$$
j^{k} a_{1}(x, y)=j^{k-1} a_{2}(x, y)=0, \quad c_{2}=\cdots=c_{k}=0 .
$$

To apply the linearization result of [3], it is necessary that

$$
j^{k} a_{1}(x, y)=j^{k-1} a_{2}(x, y)=c_{k}=0 \quad \text { or } \quad c_{2}=\cdots=c_{k}=0 .
$$

On the other hand, it follows from Theorem 3.4 that $X$ is linearizable if

$$
c_{2}=\cdots=c_{k}=0
$$

or

$$
\begin{aligned}
& (1, r) \cdot\left(i_{1}-1, i_{2}\right) \leq k-r, \quad i_{1}+i_{2} \leq k \Longrightarrow a_{1}^{\left(i_{1}, i_{2}\right)}=0, \\
& (1, r) \cdot\left(i_{1}, i_{2}-1\right) \leq k-r, \quad i_{1}+i_{2} \leq k \Longrightarrow a_{2}^{\left(i_{1}, i_{2}-1\right)}=0,
\end{aligned}
$$

where $r$ is the smallest $i$ for which $c_{i} \neq 0$. 
6.2. $\lambda=(1,0)$. The resonant monomials are $\left\{x y^{i} e_{1}, y^{i} e_{2}, i \in \mathbb{N}\right\} . \mathrm{A}$ vector field is linearizable (in the formal, real analytic, holomorphic categories) if it is of one of the two following forms:

$$
\begin{aligned}
& X(x, y)=\left(x+x^{2} \varphi(x, y), x \psi(x, y)\right) \\
& X(x, y)=\left(x+\varphi_{1}(y), 0\right) .
\end{aligned}
$$

6.3. $\lambda=(1,-k), k \in \mathbb{N}$. The resonant monomials are $\left\{x^{s k+1} y^{s} e_{1}, x^{s k} y^{s+1} e_{2}\right.$, $s \in \mathbb{N}\}$. A vector field $X$ is linearizable (in the formal, real analytic, holomorphic categories) if, for instance, it is of one of the two following forms:

$$
\begin{aligned}
& X(x, y)=\left(x+x^{2} \varphi\left(x, x^{k} y\right),-k y+\psi\left(x, x^{k} y\right)\right) \\
& X(x, y)=\left(x+\varphi\left(x^{k} y, y\right),-k y+y^{2} \psi\left(x^{k} y, y\right)\right) .
\end{aligned}
$$

\section{Applichtions: Vector Fields in $\mathbb{R}^{3}$}

In all cases considered below the vector fields and eigenvalues are real; we take as $\mathfrak{G}$ all resonant monomials that belong to the image of $L_{A}$; the monomials are represented by points in $\mathbb{Z}^{n}$, the set $\mathcal{G}$, and we construct a vector $\mu \in \mathbb{R}^{n}$ such that:

- $\mathfrak{G}$ is exactly the subset of resonant monomials for which $\mu^{*}$, the inner product with $\mu$, is strictly larger than $c \geq 0$ on $\mathcal{G}$.

- $\mathfrak{U}$ is a subset of resonant monomials for which $\mu^{*}$ is larger than or equal to $c$ on $\mathcal{U}$.

In many cases $\mathfrak{U}$ will be the set of resonant monomials for which $\mu^{*} \geq c$. Abusing notation, we can consider the value of $\mu^{*}$ on the monomial $x^{K} e_{i}$ as being $\mu \cdot P_{i}^{K}=$ $\mu \cdot\left(K-e_{i}\right)$, and similarly for $\lambda^{*}$.

It will be necessary to show that $L_{A}(\mathfrak{U})_{\mathbb{R}}=\mathfrak{G}_{\mathbb{R}}$ (it is a straightforward but often very laborious computation that we will generally omit), but the other property, $\mathcal{G}+\mathcal{U} \subset \mathcal{G}$, will follow immediately:

$$
\mu \cdot \mathcal{G}>c, \mu \cdot \mathcal{U} \geq c \Longrightarrow \mu \cdot(\mathcal{G}+\mathcal{U})>c \Longrightarrow \mathcal{G}+\mathcal{U} \subset \mathcal{G}
$$

Corollary 7.1. If there exists $\mu \in \mathbb{R}^{n}$ such that $\mathfrak{G}$ is exactly the subset of resonant monomials for which $\mu^{*}>c \geq 0$, and $\mathfrak{U}$ that for which $\mu^{*} \geq c$, the vector field $X(x)=A x+a(x)$ is linearizable if

$$
P \in \mathcal{C}, \lambda \cdot P=0 \Longrightarrow \mu \cdot P>c .
$$

In particular, a vector field $X(x)=A x+a(x)$ is linearizable if

$$
\lambda \cdot \mathcal{A} \geq 0 \quad(\text { or } \leq 0) \text { and } P \in \mathcal{A}, \lambda \cdot P=0 \Longrightarrow \mu \cdot P>c .
$$

Remark 7.2. When dealing with complex eigenvalues, we should assume that all $\lambda \cdot \mathcal{A} \in \mathbb{C}$ are in a closed (open) semiplane defined by a line through the origin, instead of the condition $\lambda \cdot \mathcal{A} \geq 0(>0)$, and similarly for the conditions involving $\mu$. 
Here we will be concerned only with vector fields whose linear part (in the Jordan canonical form) is not diagonal, with one Jordan block of dimension two. The results for the diagonal case can be obtained as in the previous section.

7.1. $\lambda=(1,1, k \neq 1), k \in \mathbb{N}$. The resonant monomials are of the form $x^{k-i} y^{i} e_{3}, i \leq$ $k$, and as $L_{A}\left(x^{k-i} y^{i} e_{3}\right)=i x^{k-i+1} y^{i-1} e_{3}$, we see that

$$
\mu=(1,0,0), \quad \mathfrak{G}=\left\{x^{k-i} y^{i} e_{3}, i<k\right\}, \quad \mathfrak{U}=\left\{x^{k-i} y^{i} e_{3}, i \leq k\right\} .
$$

Therefore a vector field $X(x, y, z)=(x, x+y, k z)+a(x, y, z)$ will be linearizable if

$$
(0, k,-1) \notin \mathcal{C}
$$

otherwise its normal form will contain just one resonant monomial, exactly $y^{k} e_{3}$ :

$$
X(x, y, z)=\left(x, x+y, k z+a y^{k}\right) .
$$

7.2. $\lambda=(k \neq 1, k, 1), k \in \mathbb{N}$. The resonant monomials are

$$
z^{k} e_{1}, \quad z^{k} e_{2}
$$

and since $L_{A}\left(z^{k} e_{1}\right)=-z^{k} e_{2}$, we see that

$$
\mu=(0,-1,0), \quad \mathfrak{G}=\left\{z^{k} e_{2}\right\}, \quad \mathfrak{U}=\left\{z^{k} e_{1}, z^{k} e_{2}\right\} .
$$

Therefore a vector field $X(x, y, z)=(k x, x+k y, z)+a(x, y, z)$ will be linearizable if

$$
(-1,0, k) \notin \mathcal{C}
$$

otherwise its normal form will contain just one resonant monomial, exactly $z^{k} e_{1}$ :

$$
X(x, y, z)=\left(k x+a z^{k}, x+k y, z\right) .
$$

Remark 7.3. In both previous cases, it is easy to verify if a given vector field is linearizable, but it is not easy to give a general formula for a linearizable vector field. The analysis of the details is similar to that of Example 6.2.

7.3. $\lambda=(1,1,0)$. The resonant monomials are

$$
\mathfrak{R}=\left\{x z^{i} e_{1}, y z^{i} e_{1}, x z^{i} e_{2}, y z^{i} e_{2}, z^{i} e_{3} \quad i \in \mathbb{N}\right\} .
$$

We can take

$$
\mu=(1,0,0), \quad \mathfrak{G}=\left\{x z^{i} e_{2}, i \in \mathbb{N}\right\}, \quad \mathfrak{U}=\left\{x z^{i} e_{1}, y z^{i} e_{2}, i \in \mathbb{N}\right\} .
$$

Therefore a vector field $X(x, y, z)=(x, x+y, 0)+a(x, y, z)$ will be linearizable if

$$
\begin{aligned}
& \frac{\partial}{\partial x} a_{1}(0,0, z) \equiv 0, \quad \frac{\partial}{\partial y} a_{1}(0,0, z) \equiv 0, \\
& \frac{\partial}{\partial y} a_{2}(0,0, z) \equiv 0, \\
& a_{3}(0,0, z) \equiv 0 .
\end{aligned}
$$

The resonant normal form will be

$$
\left(x+x \varphi_{1}(z)+y \psi_{1}(z), x+y+y \psi_{2}(z), z \theta(z)\right)
$$

where $\varphi_{1}(0)=\psi_{1}(0)=\psi_{2}(0)=\theta(0)=0$. 
7.4. $\lambda=(1,1,-k), k \in \mathbb{N}$. The resonant monomials are

$$
\left\{x^{s k+1-i} y^{i} z^{s} e_{1}, i \leq s k+1, x^{s k+1-i} y^{i} z^{s} e_{2}, i \leq s k+1, x^{s k-i} y^{i} z^{s+1} e_{3}, i \leq s k\right\},
$$

with $s \in \mathbb{N}$. From the computation of $L_{A}$ restricted to the resonant monomials, we see that we can take $\mu=(0,-1, k)$ with

$\mathfrak{G}=\left\{x^{s k+1-i} y^{i} z^{s} e_{1}, i \leq s k-1, x^{s k+1-i} y^{i} z^{s} e_{2}, i \leq s k, x^{s k-i} y^{i} z^{s+1} e_{3}, i \leq s k-1\right\}$

and

$$
\mathfrak{U}=\mathfrak{G} \cup\left\{x y^{s k} z^{s} e_{1}, y^{s k+1} z^{s} e_{2}, y^{s k} z^{s+1} e_{3}\right\} .
$$

The resonant normal form will be

$$
\left(x+x \varphi_{1}\left(y^{k} z\right)+y \psi_{1}\left(y^{k} z\right), x+y+y \psi_{2}\left(y^{k} z\right),-k z+z \theta\left(y^{k} z\right)\right),
$$

where $\varphi_{1}(0)=\psi_{1}(0)=\psi_{2}(0)=\theta(0)=0$.

7.5. $\lambda=(k \neq 1, k,-1), k \in \mathbb{N}$. The resonant monomials are

$$
\left\{x^{s+1-i} y^{i} z^{s k} e_{1}, i \leq s+1, x^{s+1-i} y^{i} z^{s k} e_{2}, i \leq s+1, x^{s-i} y^{i} z^{s k+1} e_{3}, i \leq s\right\},
$$

with $s \in \mathbb{N}$. Again from the computation of $L_{A}$ restricted to the resonant monomials, we see that we can take $\mu=(0,-k, 1)$ with

$$
\mathfrak{G}=\left\{x^{s+1-i} y^{i} z^{s k} e_{1}, i \leq s-1, x^{s+1-i} y^{i} z^{s k} e_{2}, i \leq s, x^{s-i} y^{i} z^{s k+1} e_{3}, i \leq s-1\right\}
$$

and

$$
\mathfrak{U}=\mathfrak{G} \cup\left\{x y^{s} z^{s k} e_{1}, y^{s+1} z^{s k} e_{2}, y^{s} z^{s k+1} e_{3}\right\} .
$$

The resonant normal form will be

$$
\left(k x+x \varphi_{1}\left(y z^{k}\right)+y \psi_{1}\left(y z^{k}\right), x+k y+y \psi_{2}\left(y z^{k}\right),-z+z \theta\left(y z^{k}\right)\right),
$$

where $\varphi_{1}(0)=\psi_{1}(0)=\psi_{2}(0)=\theta(0)=0$.

Remark 7.4. It is not easy, and perhaps not very useful, to give a general formula for a linearizable vector field in the two previous cases. But it is easy to see that a vector field $X(x, y, z)=(k x, x+k y,-z)+a(x, y, z)$ will be linearizable if, for instance,

$$
\begin{aligned}
& a_{1}(x, y, z)=P_{1}(x, y) \alpha_{1}\left(x, y, x z^{k}, y z^{k}\right)+x^{2} z^{k} \beta_{1}\left(x z^{k}, y z^{k}\right), \\
& a_{2}(x, y, z)=P_{2}(x, y) \alpha_{2}\left(x, y, x z^{k}, y z^{k}\right)+x \beta_{2}\left(x z^{k}, y z^{k}\right), \\
& a_{3}(x, y, z)=(a x+b y) \alpha_{3}\left(x, y, x z^{k}, y z^{k}\right)+x z^{k+1} \beta_{3}\left(x z^{k}, y z^{k}\right),
\end{aligned}
$$

where $P_{i}$ are homogeneous polynomials of degree two. Note that it contains resonant monomials, all in $\mathfrak{G}$.

7.6. $\lambda=(0,0,1)$. The resonant monomials are

$$
\left\{x^{i} y^{j} e_{1}, x^{i} y^{j} e_{2}, x^{i} y^{j} z e_{3} \quad i, j \geq 0\right\} .
$$

We can take $\mu=(1,0,0)$ with

$$
\mathfrak{G}=\left\{x^{2+i} y^{j} e_{1}, \quad x^{1+i} y^{j} e_{2}, \quad x^{1+i} y^{j} z e_{3}, \quad i, j \geq 0\right\}
$$

and $\mathfrak{U}=\mathfrak{G} \cup\left\{x y^{j} e_{1}, y^{j} e_{2}, y^{j} z e_{3}\right\}$. Therefore a vector field $X(x, y, z)=(0, x, z)+$ $a(x, y, z)$ will be linearizable if

$$
\begin{aligned}
& a_{1}(x, y, z)=x^{2} \alpha_{1}(x, y)+z \beta_{1}(x, y, z), \\
& a_{2}(x, y, z)=x \alpha_{2}(x, y)+z \beta_{2}(x, y, z), \\
& a_{3}(x, y, z)=x \alpha_{3}(x, y)+z^{2} \beta_{3}(x, y, z) .
\end{aligned}
$$


The resonant normal form will be

$$
\left(x \varphi_{1}(y)+y \psi_{1}(y), x+y \psi_{2}(y), z+z \theta(y)\right),
$$

where $\varphi_{1}(0)=\psi_{1}(0)=\psi_{2}(0)=\theta(0)=0$.

Remark 7.5. In $\mathbb{R}^{2}$, with zero a double eigenvalue and nonzero linear part, the vector field $X(x, y)=\left(x^{2} \alpha_{1}(x, y), x+x \alpha_{2}(x, y)\right)$ is linearizable and the resonant normal form is $\left(x \varphi_{1}(y)+y \psi_{1}(y), x+y \psi_{2}(y)\right)$, where $\varphi_{1}(0)=\psi_{1}(0)=\psi_{2}(0)=0$.

7.7. $\lambda=(0,0,0)$. All monomials are resonant; we assume there are two Jordan blocks, thus one of dimension two.

We can take $\mu=(1,0,0)$ with

$$
\mathfrak{G}=\left\{x^{i+2} y^{j} z^{k} e_{1}, x^{i+1} y^{j} z^{k} e_{2}, x^{i+1} y^{j} z^{k} e_{3}, i, j, k \geq 0\right\}
$$

and $\mathfrak{U}=\left\{x^{i+1} y^{j} z^{k} e_{1}, x^{i} y^{j} z^{k} e_{2}, x^{i} y^{j} z^{k} e_{3}, i, j, k \geq 0\right\}$.

A vector field is linearizable if it is of the form

$$
X(x, y, z)=\left(x^{2} \alpha_{1}(x, y, z), x+x \alpha_{2}(x, y, z), x \alpha_{3}(x, y, z)\right)
$$

and the resonant normal form is

$$
X(x, y, z)=\left(x \varphi_{1}(y, z)+\psi_{1}(y, z), x+\psi_{2}(y, z), \psi_{3}(y, z)\right)
$$

with $\varphi_{1}(0)=\psi_{1}(0)=\psi_{2}(0)=\psi_{3}(0)=0$.

\section{ACKNOWLEDGMENT}

The author profited very much from conversations with A. Davydov, V. Zakalyukin and especially M. Zhitomirskii, and he is very grateful for their comments and advice.

\section{REFERENCES}

1. D. Anosov, V. Arnold, Dynamical Systems I, Encyclopædia of Mathematical Sciences, Springer-Verlag, 1988. MR970793 (89g:58060)

2. V. Arnold, Geometrical Methods in the Theory of Ordinary Differential Equations, SpringerVerlag, 1983. MR695786 (84d:58023)

3. J. Basto-Gonçalves, I. Cruz, Analytic linearizability of some resonant vector fields, Proc. AMS 129 (2001), 2473-2481. MR1823934 (2002e:37070)

4. J. Basto-Gonçalves, A.C. Ferreira, Normal forms and linearization of vector fields with multiple eigenvalues, J. Math. Anal. Appl. 301 (2005), 219-236. MR2105931 (2005h:37104)

5. G. Belitskii, Smooth equivalence of germs of $\mathbf{C}^{\infty}$ vector fields with one zero or a pair of pure imaginary eigenvalues, Funct. Anal. Appl. 20 (1986), 253-259. MR878039 (88f:58125)

6. A. Brjuno, Analytical form of differential equations, Trans. Moscow Math. Soc. 25 (1971), 131-288. MR0377192 (51:13365)

7. C. Camacho, N. Kuiper, J. Palis, The topology of holomorphic flows with singularity, Publ. Math. IHES 48 (1978), 5-38. MR.516913 (80j:58045)

8. M. Chaperon, $C^{k}$-conjugacy of holomorphic flows near a singularity, Publ. Math. IHES 64 (1986), 693-722

9. K. T. Chen, Equivalence and decomposition of vector fields about an elementary critical point, Am. J. Math. 85 (1963), 693-722. MR0160010 (28:3224)

10. J. Guckenheimer, Hartman's theorem for complex flows in the Poincaré domain, Comp. Mathematica 24 (1972), 75-82. MR0301765 (46:920)

11. F. Ichikawa, Finitely determined singularities of formal vector fields, Inv. Math 66 (1982), 199-214. MR656620 (83j:58021)

12. G. Sell, Smooth linearization near a fixed point, Am. J. Math. 107 (1985), 1035-1091. MR $805804(87 \mathrm{c}: 58095)$

13. S. Sternberg, On the structure of local homeomorphisms of Euclidean n-space II, Am. J. Math. 80 (1958), 623-631. MR0096854 (20:3336) 
14. J. Yang, Polynomial normal forms of vector fields, Ph.D. thesis, Technion - Israel Institute of Technology, 1997.

15. _ Polynomial normal forms for vector fields on $\mathbb{R}^{3}$, Duke Math. J. 106 (2001), 1-18. MR:1810364 (2001m:34165)

16. M. Zhitomirskii, Smooth Local Normal Forms for Vector Fields and Diffeomorphisms, lecture notes, 1992.

Centro de Matemática, Universidade do Porto, Rua do Campo Alegre, 687, 4169-007 Porto, Portugal

E-mail address: jbg@fc.up.pt 